\title{
Radiologic Comparison of Humeral Position according to the Implant Designs Following Reverse Shoulder Arthroplasty: Analysis between Medial Glenoid/Medial Humerus, Lateral Glenoid/Medial Humerus, and Medial Glenoid/Lateral Humerus Designs
}

\author{
Nam Su Cho ${ }^{\bowtie}$, Ju Hyun Nam, Se Jung Hong, Tae Wook Kim, Myeong Gu Lee, Jung Tae Ahn, Yong Girl Rhee \\ Shoulder \& Elbow Clinic, Department of Orthopaedic Surgery, College of Medicine, Kyung Hee University, Seoul, Korea
}

\begin{abstract}
Background: The currently available reverse shoulder arthroplasty (RSA) designs can be classified into medial glenoid/medial humerus $(\mathrm{MGMH})$, lateral glenoid/medial humerus (LGMH), and medial glenoid/lateral humerus (MGLH) prosthesis designs. The purpose of this study was to radiologically analyze the effect of different RSA designs on humeral position following RSA.

Methods: A total of 50 patients who underwent primary RSA were retrospectively analyzed. Among 50 patients, 33 patients (group A: MGMH) underwent RSA with Aequalis system (Wright, Inc, Bloomington, MN, USA), 6 (group B: LGMH) with Aequalis system using bony increased offset, and 11 (group C: MGLH) with Aequalis Ascend Flex system. The acromiohumeral distance, acromioepiphyseal distance (AED), lateral humeral offset ( $\mathrm{LHO}), \mathrm{LHO}$ from the center of rotation $\left(\mathrm{LHO}^{\mathrm{COR}}\right)$, and deltoid length were radiologically measured to quantify the distalization and lateralization of the humerus.

Results: The increment in postoperative AED was $19.92 \pm 3.93 \mathrm{~mm}$ in group A, $24.52 \pm 5.25 \mathrm{~mm}$ in group B, and $25.97 \pm 5.29 \mathrm{~mm}$ in group $C$, respectively $(p=0.001)$. The increment in postoperative LHO was $0.13 \pm 6.30 \mathrm{~mm}, 8.00 \pm 12.14 \mathrm{~mm}$, and $7.42 \pm 6.88$ $\mathrm{mm}$, respectively $(p=0.005)$. The increment in postoperative $\mathrm{LHO}^{\mathrm{COR}}$ was $20.76 \pm 6.06 \mathrm{~mm}, 22.04 \pm 5.15 \mathrm{~mm}$, and $28.11 \pm 4.14$ $\mathrm{mm}$, respectively $(p=0.002)$.

Conclusions: The radiologic analysis of the effect of different RSA designs on humeral position following RSA showed significant differences in the increment in postoperative AED, LHO, and LHO ${ }^{\mathrm{CO}}$ between the 3 groups. Therefore, MGLH design seems to be more effective for humeral distalization and lateralization compared to original Grammont design.
\end{abstract}

(Clin Shoulder Elbow 2018;21(4):192-199)

Key Words: Shoulder; Cuff tear arthropathy; Massive rotator cuff tear; Reverse shoulder arthroplasty; Implant design

\section{Introduction}

The Grammont prosthetic design of reverse shoulder arthroplasty (RSA) is widely used in irreparable rotator cuff deficient patients or cuff tear arthropathy patients, and provides postoperative pain relief and improved function. ${ }^{1,2)}$ Numerous recent studies have reported positive clinical outcomes of RSA. ${ }^{1,3-5)}$ However, several complications or adverse effects have also been reported, including scapular notching, acromial fracture, glenoid loosening, instability, neurologic lesions and excessive arm lengthening. ${ }^{3,6-8)}$ To overcome these complications, some modifications in the prosthesis and surgical techniques of the Grammont design have been proposed: bony lateralization or metallic lateralization of the center of rotation (COR); different baseplate implantation techniques; modification of the glenoshpere shape; and modification of the neck-shaft angle of the

Received June 10, 2018. Revised July 27, 2018. Accepted July 30, 2018.

Correspondence to: Nam Su Cho

Department of Orthopaedic Surgery, Kyung Hee University Hospital at Gangdong, College of Medicine, Kyung Hee University, 892 Dongnamro, Gangdong-gu, Seoul 05278, Korea

Tel: +82-2-440-6154, Fax: +82-2-440-7498, E-mail: nscos1212@empas.com, ORCID: http://orcid.org/0000-0003-1643-1641

IRB approval: Kyung Hee University Hospital at Gangdong (KHNMC 2018-06-010).

Financial support: None. Conflict of interests: None. 
humerus. ${ }^{9-14)}$ Based on the different prosthesis combinations of glenoid and humeral offsets, the current available RSA designs are classified as medial glenoid/medial humerus (MGMH), lateral glenoid/medial humerus (LGMH), and medial glenoid/lateral humerus (MGLH) prosthesis. ${ }^{15)}$

The MGMH design, such as the Grammont prosthesis, achieves an increased deltoid moment arm and reduces shear forces at the fixation surface by medializing the COR to the face of the scapula. ${ }^{3)}$ The LGMH design is characterized by more lateral glenoid position and more medial humeral position, allowing for better residual rotator cuff tension and improved deltoid wrapping. ${ }^{16-18)}$ However, due to the more lateralized COR, the deltoid abductor moment arm of the LGMH design is less than the MGMH designs. ${ }^{12,19,20)}$ Furthermore, there is an increased risk of baseplate failure and glenoid loosening due to stress concentration and shear forces on the bone-implant surface of the glenoid component. ${ }^{12,19-22)}$

The MGLH design allows the lateral position of the humerus to compensate for the relative medialization of the COR due to the medial glenoid position. ${ }^{15)}$ The lateral position of the humerus allows better tensioning of the residual rotator cuff, greater deltoid wrapping, and increased deltoid abductor moment arms as compared with the original Grammont design. Additional advantages of this technique include reduction of scapular notching, lower glenoid loosening rate, and improved range of motion (ROM). ${ }^{15}$

Since each design has its own advantages and disadvantages, the debate to decide the most suitable RSA design continues. To date, the impacts of RSA design on postoperative humeral position are not been well-studied. This study was therefore undertaken to radiologically analyze the effect of different RSA designs on humeral position following RSA. Considering the characteristics of all three designs, we hypothesized that there would be differences between the 3 groups (the MGLH group, LGMH group, and MGMH group) for lateral humeral offset (LHO), and the MGLH group would have greater $\mathrm{LHO}$ from the $\mathrm{COR}\left(\mathrm{LHO}^{\mathrm{COR}}\right)$.

\section{Methods}

Final approval of exemption from review by the Institutional Review Board was obtained for this study due to its retrospective nature (KHNMC 2018-06-010).

\section{Patient Selection}

Totally, 57 shoulders (57 patients) that underwent primary RSA for irreparable rotator cuff tear or cuff tear arthropathy between September 2009 and April 2017 were enrolled for this study and retrospectively analyzed. Patients preoperatively diagnosed as glenohumeral arthritis (1 case), secondary glenohumeral arthritis due to infection sequelae or trauma (2 cases), four-part proximal humerus fracture (3 cases), or osteonecrosis of humeral head ( 1 case) were excluded from the study. Finally, a total of 50 patients ( 10 male and 40 female) were included in this study. The mean patient age at the time of operation was 75.3 years (range, $64-86$ years).

Of the 50 patients included in the final analysis, 33 patients (group A: MGMH) underwent RSA using the Aequalis system (Wright, Inc., Bloomington, MN, USA), 6 patients (group B: LGMH) with Aequalis system using bony increased offset (Wright, Inc.), and 11 patients (group C: MGLH) with Aequalis Ascend Flex system (Wright, Inc.).

\section{Preoperative and Postoperative Evaluations}

All patients underwent preoperative and postoperative radiologic examinations. All radiographic measurements were performed on a true anteroposterior view of each patient. The acromiohumeral distance (AHD) (Fig. 1), acromioepiphyseal distance (AED), $\mathrm{LHO}, \mathrm{LHO}^{\mathrm{COR}}$, and deltoid length were radiologically measured to quantify the distalization and lateralization of the humerus. All parameters were evaluated twice by a single observer to assess repeatability of the measurements. Intraobserver reproducibility was based on the data acquired during the measurement series.

The distance between the most lateral point of the under-
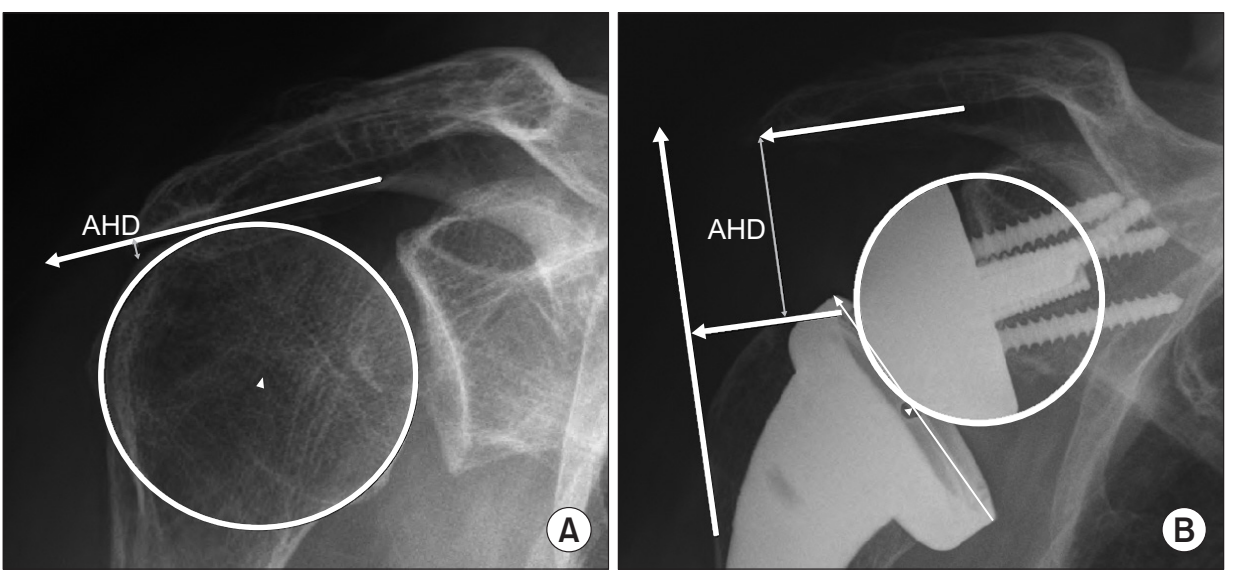

Fig. 1. Preoperative (A) and postoperative (B) acromiohumeral distance (AHD). 
surface of the acromion and a line parallel to the top of the greater tuberosity was defined as the AHD (Fig. 2). ${ }^{23)}$ As previously described by Renaud et al., ${ }^{24)}$ the distance between a line parallel to the superior cortex of the acromion and the COR of the humeral head or the center of the prosthetic epiphysis was measured as the AED (Fig. 3). The LHO was determined as the distance from the perpendicular line to the most lateral point of the undersurface of the acromion to the most lateral part of the greater tuberosity (Fig. 4). ${ }^{23)}$ Humeral offset from the COR of humeral head or from the glenosphere to the most lateral point of greater tuberosity was measured as the $\mathrm{LHO}^{\mathrm{COR}}$, as described previously by Lädermann et al. ${ }^{25)}$ (Fig. 4). The distance from the inferolateral tip of the acromion to the midpoint of the deltoid tuberosity $^{26)}$ was defined as the deltoid length (Fig. 5), as initially
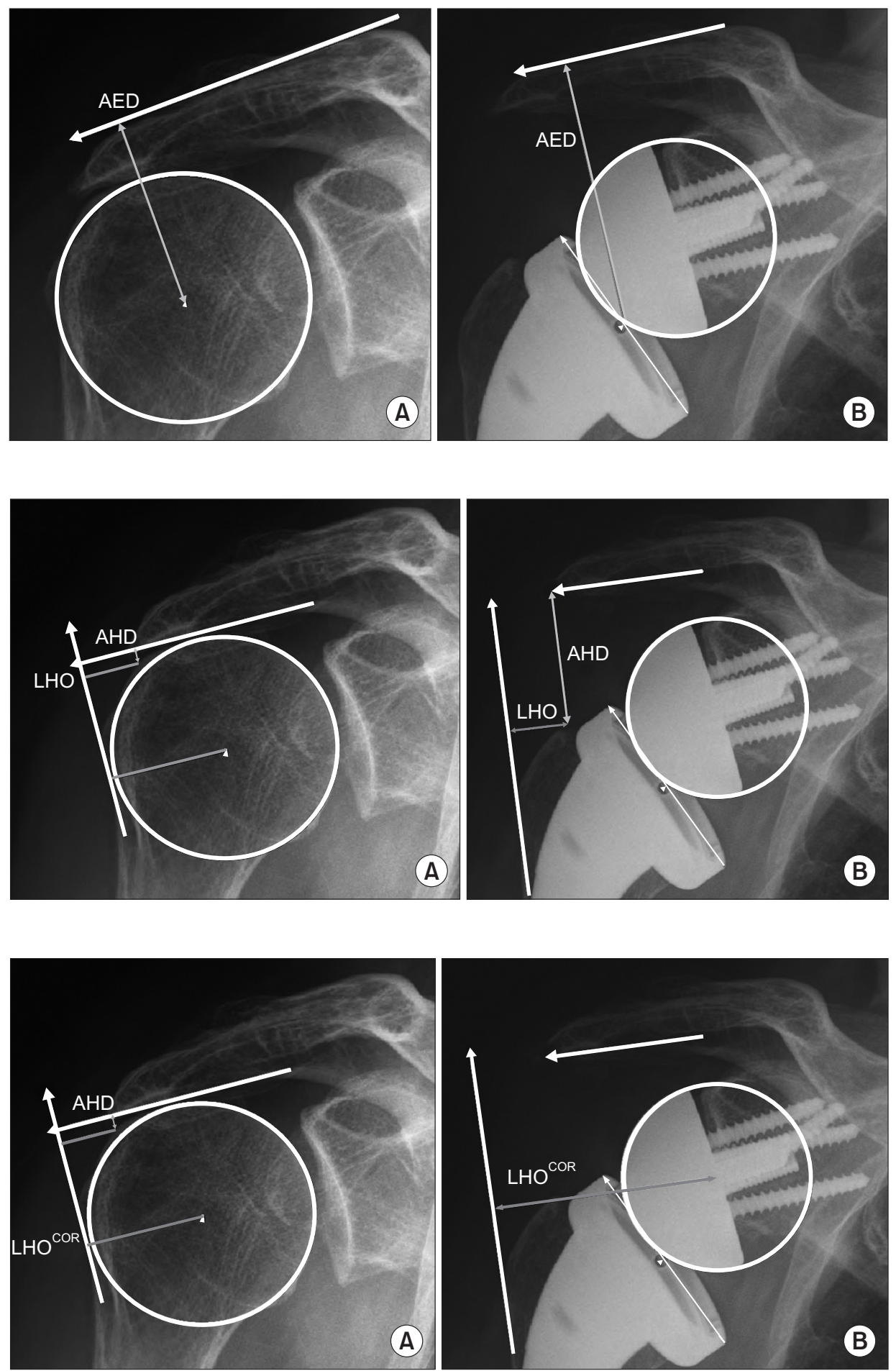

Fig. 4. Preoperative (A) and postoperative (B) lateral humeral offset from the center of rotation $\left(\mathrm{LHO}^{\mathrm{COR}}\right)$.

AHD: acromiohumeral distance. 

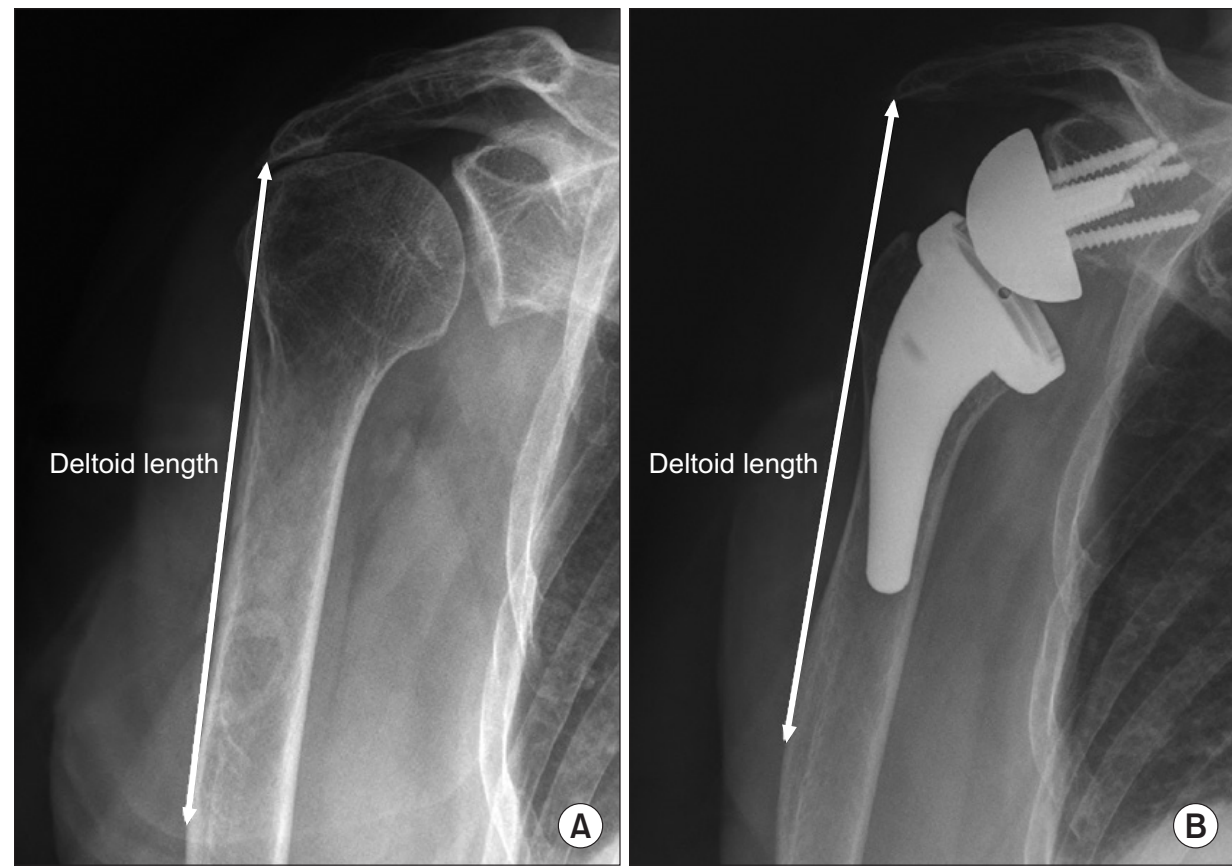

Fig. 5. Preoperative (A) and postoperative (B) deltoid length.

described by De Wilde et al. ${ }^{27)}$ All measurements were assessed preoperatively and postoperatively.

\section{Operative Techniques}

All operations were performed by the senior author; patient was under general anesthesia and placed in a beach chair flexed about $30^{\circ}$. For prosthesis, the Aequalis system (Wright, Inc.) was used for 33 of the 50 patients, the Aequalis system using bonyincreased offset (Wright, Inc.) was used for 6 patients, and the Aequalis Ascend Flex system (Wright, Inc.) was used for 11 patients. In all patients, implantation of the prosthesis was achieved using the deltopectoral approach. Remnants of the subscapularis tendon were detached during exposure and tagging sutured. For the Aequalis system using bony-increased offset, a $10 \mathrm{~mm}$ thick humeral head bone block was harvested using a cutting guide for bone graft, to lateralize the COR. The glenoid baseplate was tilted $5^{\circ}$ to $10^{\circ}$ inferiorly, and the center of glenoid ball was targeted just posterior and inferior to the bare spot to minimize scapular notching in all patients.

Tension of the deltoid muscle and conjoined tendon was checked with a trial cup; both tensions were checked again during distal humeral traction with a real implant. If possible, the detached subscapularis tendon was repaired. After wound closure, the upper extremity was placed in a 'hand shake' orthosis.

\section{Statistical Analysis}

The Wilcoxon signed-rank test was used for data analysis between the preoperative and postoperative measurements of each group. Kruskal-Wallis test with a Tukey post hoc comparison analyzed the data between the three groups. Significance was set at a level of 0.05 with associated $95 \%$ confidence intervals. The SPSS software package ver. 16.0 (SPSS Inc., Chicago, IL, USA) was used for all statistical analyses.

\section{Results}

The intra-observer correlation coefficient of repeated radiographic measurements ranged from 0.979 to 0.998 for single measurements, and from 0.988 to 0.999 for average measurements. The results demonstrate good to excellent intra-observer reproducibility, indicating that all measurements can be performed with good repeatability.

Groups A and B showed statistically significant postoperative increases for all factors except postoperative LHO (Table 1 and Table 2, respectively), whereas Group $C$ showed significant postoperative increases for all factors (Table 3). There were no statistical differences in preoperative radiologic measurements of each item when comparing the three groups, but postoperative measurements of $\mathrm{AED}, \mathrm{LHO}$, and $\mathrm{LHO}^{\mathrm{COR}}$ showed statistical differences among the three groups (Table 4).

The increment in postoperative AHD was $23.70 \pm 5.88$ $\mathrm{mm}$ in group $\mathrm{A}, 28.12 \pm 6.01 \mathrm{~mm}$ in group $\mathrm{B}$, and $27.90 \pm$ $8.49 \mathrm{~mm}$ in group $C$, with no statistical difference observed between the three groups ( $p=0.101$ ) (Table 5). The increment in postoperative AED was $19.92 \pm 3.93 \mathrm{~mm}$ in group $\mathrm{A}, 24.52$ $\pm 5.25 \mathrm{~mm}$ in group $B$, and $25.97 \pm 5.29 \mathrm{~mm}$ in group $C$, and was significantly different between the groups $(p=0.001)$. The increment in postoperative LHO was $0.13 \pm 6.30 \mathrm{~mm}, 8.00 \pm$ $12.14 \mathrm{~mm}$, and $7.42 \pm 6.88 \mathrm{~mm}$, respectively, with statistically significant differences $(p=0.005)$. The increment in postopera- 
Clinics in Shoulder and Elbow

Vol. 21, No. 4, December, 2018

Table 1. Group A (MGMH)

\begin{tabular}{|c|c|c|c|c|c|}
\hline Variable & AHD & AED & LHO & $\mathrm{LHO}^{\mathrm{COR}}$ & Deltoid length \\
\hline Preoperative (mm) & 5.05 & 33.82 & 14.56 & 22.73 & 147.96 \\
\hline Postoperative (mm) & 28.75 & 53.75 & 14.68 & 43.49 & 169.13 \\
\hline Difference $(\mathrm{mm})$ & 23.70 & 19.92 & 0.13 & 20.76 & 21.18 \\
\hline$p$-value & $<0.001$ & $<0.001$ & 0.909 & $<0.001$ & $<0.001$ \\
\hline
\end{tabular}

Values are presented as mean only.

MGMH: medial glenoid/medial humerus, AHD: acromiohumeral distance, AED: acromioepiphyseal distance, LHO: lateral humeral offset, LHO ${ }^{\text {COR }}$ : lateral humeral offset from the center of rotation.

Table 2. Group B (LGMH)

\begin{tabular}{|c|c|c|c|c|c|}
\hline Variable & AHD & AED & LHO & $\mathrm{LHO}^{\mathrm{COR}}$ & Deltoid length \\
\hline Preoperative (mm) & 3.19 & 33.12 & 13.24 & 21.27 & 147.55 \\
\hline Postoperative (mm) & 31.31 & 57.64 & 21.24 & 43.31 & 173.45 \\
\hline Difference (mm) & 28.12 & 24.52 & 8.00 & 22.04 & 25.90 \\
\hline$p$-value & $<0.001$ & $<0.001$ & 0.167 & $<0.001$ & 0.01 \\
\hline
\end{tabular}

Values are presented as mean only.

LGMH: lateral glenoid/medial humerus, AHD: acromiohumeral distance, AED: acromioepiphyseal distance, LHO: lateral humeral offset, LHO $^{\text {COR }}$ : lateral humeral offset from the center of rotation.

Table 3. Group C (MGLH)

\begin{tabular}{lccccc}
\hline \multicolumn{1}{c}{ Variable } & AHD & AED & LHO & LHO $^{\text {COR }}$ & Deltoid length \\
\hline Preoperative $(\mathrm{mm})$ & 2.12 & 32.78 & 15.53 & 20.15 & 145.27 \\
\hline Postoperative $(\mathrm{mm})$ & 30.17 & 58.75 & 22.95 & 48.25 & 173.18 \\
Difference $(\mathrm{mm})$ & 27.90 & 25.97 & 7.42 & 28.10 & 27.91 \\
p-value & $<0.001$ & $<0.001$ & 0.005 & $<0.001$ & $<0.001$ \\
\hline
\end{tabular}

Values are presented as mean only.

MGLH: medial glenoid/lateral humerus, AHD: acromiohumeral distance, AED: acromioepiphyseal distance, LHO: lateral humeral offset, LHO ${ }^{\mathrm{COR}}$ : lateral humeral offset from the center of rotation.

Table 4. Preoperative and Postoperative Comparison among Groups A, B, and C

\begin{tabular}{|c|c|c|c|c|c|}
\hline Variable & AHD & AED & LHO & $\mathrm{LHO}^{\mathrm{COR}}$ & Deltoid length \\
\hline \multicolumn{6}{|l|}{ Preoperative } \\
\hline Group A (mm) & 5.05 & 33.82 & 14.56 & 22.73 & 145.27 \\
\hline Group B (mm) & 3.19 & 33.12 & 13.24 & 21.27 & 147.55 \\
\hline Group C (mm) & 2.12 & 32.78 & 15.53 & 20.15 & 145.27 \\
\hline$p$-value & 0.058 & 0.679 & 0.762 & 0.125 & 0.868 \\
\hline \multicolumn{6}{|l|}{ Postoperative } \\
\hline Group A (mm) & 28.75 & 53.75 & 14.68 & 43.49 & 169.13 \\
\hline Group B (mm) & 31.31 & 57.64 & 21.24 & 43.31 & 173.45 \\
\hline Group C (mm) & 30.17 & 58.75 & 22.95 & 48.25 & 173.18 \\
\hline$p$-value & 0.521 & 0.011 & 0.04 & 0.036 & 0.706 \\
\hline
\end{tabular}

Values are presented as mean only.

Group A: medial glenoid/medial humerus, Group B: lateral glenoid/medial humerus, Group C: medial glenoid/lateral humerus, AHD: acromiohumeral distance, AED: acromioepiphyseal distance, $\mathrm{LHO}$ : lateral humeral offset, $\mathrm{LHO}^{\mathrm{COR}}$ : lateral humeral offset from the center of rotation. 
Table 5. Comparison of Differences in Increment among Groups A, B, and C

\begin{tabular}{lcccccc}
\hline Variable & AHD & AED & LHO & LHO $^{\text {COR }}$ & Deltoid length \\
\hline Group A $(\mathrm{mm})$ & 23.70 & 19.92 & 0.13 & 20.76 & 21.18 \\
Group B (mm) & 28.12 & 24.52 & 8.00 & 7.42 & 28.04 & 25.90 \\
Group C $(\mathrm{mm})$ & 27.90 & 25.97 & 0.001 & 0.005 & 0.002 & 0.095 \\
\hline -value & 0.101 & & & \\
\hline
\end{tabular}

Values are presented as mean only.

Group A: medial glenoid/medial humerus, Group B: lateral glenoid/medial humerus, Group C: medial glenoid/lateral humerus, AHD: acromiohumeral distance, AED: acromioepiphyseal distance, LHO: lateral humeral offset, $\mathrm{LHO}^{\mathrm{COR}}$ : lateral humeral offset from the center of rotation.

Table 6. Multiple Comparisons

\begin{tabular}{|c|c|c|c|c|c|}
\hline Dependent variable & Group & & $p$-value & & \\
\hline \multirow[t]{6}{*}{$\triangle \mathrm{AED}$} & A & $\mathrm{B}$ & 0.062 & -9.38 & 0.19 \\
\hline & & $\mathrm{C}$ & 0.001 & -9.80 & -2.30 \\
\hline & $\mathrm{B}$ & $\mathrm{A}$ & 0.062 & -0.19 & 9.38 \\
\hline & & $\mathrm{C}$ & 0.798 & -6.92 & 4.02 \\
\hline & $\mathrm{C}$ & $\mathrm{A}$ & 0.001 & 2.27 & 9.80 \\
\hline & & $\mathrm{B}$ & 0.798 & -4.02 & 6.92 \\
\hline \multirow[t]{6}{*}{$\Delta \mathrm{LHO}$} & A & $\mathrm{B}$ & 0.047 & -15.68 & -0.08 \\
\hline & & $\mathrm{C}$ & 0.016 & -13.41 & -1.17 \\
\hline & $\mathrm{B}$ & A & 0.047 & 0.08 & 15.68 \\
\hline & & $\mathrm{C}$ & 0.986 & -8.34 & 9.50 \\
\hline & $\mathrm{C}$ & A & 0.016 & 1.17 & 13.41 \\
\hline & & $\mathrm{B}$ & 0.986 & -9.50 & 8.34 \\
\hline \multirow[t]{6}{*}{$\Delta \mathrm{LHO}^{\mathrm{COR}}$} & A & B & 0.864 & -7.31 & 4.74 \\
\hline & & $\mathrm{C}$ & 0.001 & -12.07 & -2.62 \\
\hline & B & A & 0.864 & -4.74 & 7.31 \\
\hline & & $\mathrm{C}$ & 0.094 & -12.96 & 0.82 \\
\hline & $\mathrm{C}$ & $\mathrm{A}$ & 0.001 & 2.62 & 12.07 \\
\hline & & $\mathrm{B}$ & 0.094 & -0.82 & 12.96 \\
\hline
\end{tabular}

Group A: medial glenoid/medial humerus, Group B: lateral glenoid/medial humerus, Group C: medial glenoid/lateral humerus, CI: confidence interval, $\Delta$ : postoperative increment, AED: acromioepiphyseal distance, LHO: lateral humeral offset, $\mathrm{LHO}^{\mathrm{COR}}$ : lateral humeral offset from the center of rotation.

tive $\mathrm{LHO}^{\mathrm{COR}}$ was $20.76 \pm 6.06 \mathrm{~mm}$ in group $\mathrm{A}, 22.04 \pm 5.15$ $\mathrm{mm}$ in group $\mathrm{B}$, and $28.11 \pm 4.14 \mathrm{~mm}$ in group $\mathrm{C}$, and was also statistically significant $(p=0.002)$. The increments in postoperative deltoid length for groups $\mathrm{A}, \mathrm{B}$ and $\mathrm{C}$ were $21.18 \pm 7.27 \mathrm{~mm}$, $25.90 \pm 15.61 \mathrm{~mm}$, and $27.91 \pm 10.51 \mathrm{~mm}$, respectively, but were not statistically significant $(p=0.095)$. The multiple comparisons revealed no statistically significant differences between group $\mathrm{B}$ and $\mathrm{C}$, but there were significant differences between groups $A$ and $B$ in the increment of $\mathrm{LHO}$, and between groups $A$ and $C$ in increments of $\mathrm{AED}, \mathrm{LHO}$, and $\mathrm{LHO}^{\mathrm{COR}}$ (Table 6).

\section{Discussion}

The impacts of RSA design on postoperative humeral position have not been well-studied. Also, it is uncertain whether the theoretical effects of MGLH design can be confirmed by radiologic imaging. Moreover, some studies have reported the results of each design individually, but, to our knowledge, no clinical study has compared the radiologic measurements of all three designs simultaneously. Therefore, this study was undertaken to evaluate the imaging data obtained from surgeries performed using all three designs, all executed by a single surgeon.

Numerous biomechanical studies have recently reported efficacy of the MGLH design. Lädermann et al. ${ }^{25)}$ reported that 
the $145^{\circ}$ onlay stem was more effective than the traditional Grammont design in ROM. Hamilton et al. ${ }^{28)}$ reported that the MGLH design showed the highest efficiency compared to the MGMH and LGMH designs, and had the greatest posterior deltoid moment arm. A biomechanical study by Routman et al. ${ }^{15)}$ showed that the MGLH design had a lower scapular notching rate compared to the MGMH design. Another biomechanical study found the MGLH design has a large humeral offset and the greatest moment arm during external rotation. ${ }^{16)}$ A recently published clinical study further reported the clinical success of the MGLH design. Friedman et al. ${ }^{29)}$ demonstrated that the lateralized humeral prosthesis showed a lower rate of instability and dislocation and better clinical improvement in ROM and outcome scores. ${ }^{29)}$

Since the concept of MGLH design is ideal and several studies have published biomechanical and clinical advantages of this design, ${ }^{15,19)}$ we assumed that MGLH would have a clear radiological difference based on its design philosophy compared to other designs. However, in this study, the radiologic analysis of the effect of different RSA designs on humeral position following RSA showed no statistically significant differences in increment values among the three groups in postoperative AHD. This result differs from the findings of Lädermann et al. ${ }^{25)}$ who observed that the increment in postoperative AHD of $145^{\circ}$ onlay MGLH design group was shorter than the traditional $155^{\circ}$ inlay MGMH Grammont design group. Unlike our hypothesis, there were significant differences in the increment of AED among the three groups when considering increment of $\mathrm{LHO}$ and $\mathrm{LHO}^{\mathrm{COR}}$, but no statistically significant difference for the increment in postoperative deltoid length. In multiple comparisons, the MGLH group showed significant differences in the increment in postoperative $\mathrm{AED}, \mathrm{LHO}$ and $\mathrm{LHO}^{\mathrm{COR}}$ compared to the $\mathrm{MGMH}$ group, but showed no significant differences compared to the LGMH group. The LGMH group showed significant difference in increment of $\mathrm{LHO}$ compared to the MGMH group. This is consistent with the findings of Lädermann et al., ${ }^{25)}$ who reported greater increment in postoperative $\mathrm{LHO}$ of $145^{\circ}$ onlay MGLH design than traditional $155^{\circ}$ inlay MGMH Grammont design. As the position of the eccentric tray (which is expected to make theoretically diverse differences) does not actually have a significant effect on arm lengthening in the study of Werner et al. ${ }^{30)}$ the values of some factors differed in the results of this study as compared to our hypotheses. This is probably because the concept or philosophy of each design does not directly make a significant difference in the imaging measurements.

Our study has a few limitations. First, being retrospective in nature, our study has limitations similar to other retrospective studies. Second, the number of patients included in this study is small. Only 6 patients were included in group B. In addition, although both bony-increased offset and metallic offset can be used for RSA using the LGMH design, this study used only the bony-increased offset. Also, a centric or an eccentric humeral tray configuration can be used for artificial joint surgery using the MGLH design, and while using the eccentric humeral tray configuration, the dial can be rotated in different directions depending on the situation of each individual case at the time of operation. Lastly, all postoperative follow-up images may not be exact true anteroposterior views in neutral rotation. However, the observer tried to measure an image as close as possible to a true anteroposterior view in neutral rotation. Minor changes in arm position or shoulder angle could affect each measurement. Despite several limitations of this study, the results of the current study can be coherent because this study is a single-surgeon series performing all three different designs of operations in the same standardized method.

\section{Conclusion}

The radiologic analysis of the effect of different RSA designs on humeral position following RSA showed no significant difference between the three groups in postoperative AHD and deltoid length increments. However, significant differences were observed in the postoperative AED, $\mathrm{LHO}$ and $\mathrm{LHO}^{\mathrm{COR}}$ increment values. In multiple comparison, the MGLH group showed significant differences in the increment in postoperative AED, $\mathrm{LHO}$ and $\mathrm{LHO}^{\mathrm{COR}}$ compared to the MGMH group, but the LGMH group showed significant difference in LHO only compared to the MGMH group. Taken together, we believe that the MGLH design is effective for humeral distalization and lateralization compared to the original Grammont design.

\section{References}

1. Wall B, Nové-Josserand L, O'Connor DP, Edwards TB, Walch G. Reverse total shoulder arthroplasty: a review of results according to etiology. J Bone Joint Surg Am. 2007;89(7):1476-85.

2. Ramirez MA, Ramirez J, Murthi AM. Reverse total shoulder arthroplasty for irreparable rotator cuff tears and cuff tear arthropathy. Clin Sports Med. 2012;31(4):749-59.

3. Boileau P, Watkinson DJ, Hatzidakis AM, Balg F. Grammont reverse prosthesis: design, rationale, and biomechanics. J Shoulder Elbow Surg. 2005;14(1 Suppl S):147S-61S.

4. Boileau P, Gonzalez JF, Chuinard C, Bicknell R, Walch G. Reverse total shoulder arthroplasty after failed rotator cuff surgery. J Shoulder Elbow Surg. 2009;18(4):600-6.

5. Drake GN, O'Connor DP, Edwards TB. Indications for reverse total shoulder arthroplasty in rotator cuff disease. Clin Orthop Relat Res. 2010;468(6):1526-33.

6. Melis B, DeFranco M, Lädermann A, et al. An evaluation of the radiological changes around the Grammont reverse geometry shoulder arthroplasty after eight to 12 years. J Bone Joint Surg Br. 2011;93(9):1240-6. 
7. Lädermann A, Edwards TB, Walch G. Arm lengthening after reverse shoulder arthroplasty: a review. Int Orthop. 2014;38(5): 991-1000.

8. Lädermann A, Williams MD, Melis B, Hoffmeyer P, Walch G. Objective evaluation of lengthening in reverse shoulder arthroplasty. J Shoulder Elbow Surg. 2009;18(4):588-95.

9. Gutiérrez S, Levy JC, Frankle MA, et al. Evaluation of abduction range of motion and avoidance of inferior scapular impingement in a reverse shoulder model. J Shoulder Elbow Surg. 2008;17(4):608-15.

10. Skikumaran U, Salari N, Garzon-Muvdi J, et al. Prosthetic design of reverse total shoulder arthroplasty contributes to scapular notching and instability. Paper presented at: 12th International Congress of Shoulder and Elbow Surgery; 2013 Apr 1013; Nagoya, Japan. p.180.

11. Trouilloud P, Gonzalvez M, Martz P, et al. Duocentric ${ }^{\circledR}$ reversed shoulder prosthesis and Personal Fit ${ }^{\circledR}$ templates: innovative strategies to optimize prosthesis positioning and prevent scapular notching. Eur J Orthop Surg Traumatol. 2014;24(4): 483-95.

12. Boileau P, Moineau G, Roussanne Y, O'Shea K. Bony increased-offset reversed shoulder arthroplasty: minimizing scapular impingement while maximizing glenoid fixation. Clin Orthop Relat Res. 2011;469(9):2558-67.

13. Boughebri O, Duparc F, Adam JM, Valenti P. Arthroscopic dynamic analysis of scapular notching in reverse shoulder arthroplasty. Orthop Traumatol Surg Res. 2011;97(8):779-84.

14. Sadoghi $P$, Leithner A, Vavken $P$, et al. Infraglenoidal scapular notching in reverse total shoulder replacement: a prospective series of 60 cases and systematic review of the literature. BMC Musculoskelet Disord. 2011;12:101.

15. Routman HD, Flurin PH, Wright TW, Zuckerman JD, Hamilton MA, Roche CP. Reverse shoulder arthroplasty prosthesis design classification system. Bull Hosp Jt Dis (2013). 2015;73 Supple 1:S5-14.

16. Hamilton MA, Roche CP, Diep P, Flurin PH, Routman HD. Effect of prosthesis design on muscle length and moment arms in reverse total shoulder arthroplasty. Bull Hosp Jt Dis (2013). 2013;71 Supple 2:S31-5.

17. Roche CP, Diep P, Hamilton M, et al. Impact of inferior glenoid tilt, humeral retroversion, bone grafting, and design parameters on muscle length and deltoid wrapping in reverse shoulder arthroplasty. Bull Hosp Jt Dis (2013). 2013;71(4):284-93.
18. Routman HD. The role of subscapularis repair in reverse total shoulder arthroplasty. Bull Hosp Jt Dis (2013). 2013;71 Suppl 2:108-12.

19. Valenti $P$, Sauzières $P$, Katz D, Kalouche I, Kilinc AS. Do less medialized reverse shoulder prostheses increase motion and reduce notching? Clin Orthop Relat Res. 2011;469(9):2550-7.

20. Middleton C, Uri O, Phillips S, et al. A reverse shoulder arthroplasty with increased offset for the treatment of cuff-deficient shoulders with glenohumeral arthritis. Bone Joint J. 2014;96B(7): 936-42.

21. Flatow EL, Harrison AK. A history of reverse total shoulder arthroplasty. Clin Orthop Relat Res. 2011;469(9):2432-9.

22. Jazayeri R, Kwon YW. Evolution of the reverse total shoulder prosthesis. Bull NYU Hosp Jt Dis. 2011;69(1):50-5.

23. Werner BS, Jacquot A, Molé D, Walch G. Is radiographic measurement of acromiohumeral distance on anteroposterior view after reverse shoulder arthroplasty reliable? J Shoulder Elbow Surg. 2016;25(9):e276-80.

24. Renaud P, Wahab H, Bontoux L, Dauty M, Richard I, Brégeon C. [Total inverted shoulder prosthesis and rotator cuff insufficiency: evaluation and determination of anatomical parameters predictive of good functional outcome in 21 shoulders]. Ann Readapt Med Phys. 2001;44(5):273-80.

25. Lädermann A, Denard PJ, Boileau P, et al. Effect of humeral stem design on humeral position and range of motion in reverse shoulder arthroplasty. Int Orthop. 2015;39(11):2205-13.

26. Jobin CM, Brown GD, Bahu MJ, et al. Reverse total shoulder arthroplasty for cuff tear arthropathy: the clinical effect of deltoid lengthening and center of rotation medialization. J Shoulder Elbow Surg. 2012;21(10):1269-77.

27. De Wilde LF, Audenaert EA, Berghs BM. Shoulder prostheses treating cuff tear arthropathy: a comparative biomechanical study. J Orthop Res. 2004;22(6):1222-30.

28. Hamilton MA, Diep P, Roche C, et al. Effect of reverse shoulder design philosophy on muscle moment arms. J Orthop Res. 2015;33(4):605-13.

29. Friedman RJ, Flurin PH, Wright TW, Zuckerman JD, Roche CP. Comparison of reverse total shoulder arthroplasty outcomes with and without subscapularis repair. J Shoulder Elbow Surg. 2017;26(4):662-8.

30. Werner BS, Ascione F, Bugelli G, Walch G. Does arm lengthening affect the functional outcome in onlay reverse shoulder arthroplasty? J Shoulder Elbow Surg. 2017;26(12):2152-7. 